\title{
Análisis clínico de 91 casos de vasculitis de mediano y pequeño vaso en adultos
}

\author{
S. GONZÁLEZ QUIJADA, M. ${ }^{a}$ SEVIL PURAS, A. R. VALLEDOR, R. LOMA \\ Secciones de Medicina Interna y de Familia. Hospital General Yagüe. Burgos
}

\author{
CLINICAL ANALYSIS OF 91 ADULTS WITH MEDIUM AND SMALL \\ SIZED VESSEL VASCULITIS
}

\section{RESUMEN}

Fundamento: Analizar las diferencias clínicas y biológicas de las vasculitis de mediano y pequeño vaso.

Pacientes y métodos: Estudio descriptivo retrospectivo de 91 casos de vasculitis atendidos en nuestro hospital entre enero de 1991 a marzo de 2001. Se describen las características clínicas y analíticas.

Resultados: El 57\% eran varones y la edad media fue de 61,9 $\pm 18,6$ años (mínima de 17 y máxima de 90 ). Los síntomas y afectación orgánica fueron: púrpura palpable $(89 \%)$, fiebre $(36 \%)$, astenia (20\%), artromialgias (19\%), afectación renal (18\%), artritis (16\%), dolor abdominal $(16 \%)$, neuropatía $(8,7 \%)$, afectación pulmonar $(6,5 \%)$. El $24 \%$ mostró varios brotes, clínica prolongada o cronificación del proceso. El 42\% mostró afectación de dos o más órganos o sistemas. Los pacientes con vasculitis consideradas pauci-inmunitarias mostraron mayor astenia $(\mathrm{p}<0,001)$, afectación renal y pulmonar $(\mathrm{p}<0,001$ y $\mathrm{p}<0,01$; respectivamente), afectación multiorgánica ( $\mathrm{p}<0,001)$ y mortalidad relacionada con el proceso $(\mathrm{p}<0,05)$. No se encontraron diferencias significativas con respecto al resto de la clínica analizada.

Conclusiones: Aunque existe un importante solapamiento clínico entre los distintos tipos de vasculitis, la presencia o ausencia de ciertos datos clínicos y biológicos son de ayuda en la diferenciación y caracterización de las distintas entidades.

PALABRAS CLAVE: Vasculitis. Análisis clínico
ABSTRACT

Objective: To evaluate the clinical and biological differences between medium sized vessel vasculitis and small vessel vasculitis.

Patients and methods: descriptive and retrospective study of 91 patients with vasculitis attended in our hospital from January 1991 to mars 2001. We describe the characteristics of clinical and analytic features.

Results: $57 \%$ were males. The mean age was $61.9 \pm 18.6$ years (17 to 90 years). The symptoms and affected organs were: palpable purpura (89\%), fever (36\%), asthenia (20\%), arthromyalgias (19\%), nephropaty (18\%), arthritis (16\%), abdominal pain (16\%), neuropathy (8,7\%), pulmonary involvement (6,5\%). 25\% had several episodes, lasting clinical, chronic disease. $42 \%$ had evidence of two or more involve organs. The patients with pauci-inmune vasculitis presented more asthenia, nephritis, pulmonary involvement, multi-organic involvement and mortality related to the process. We did not found significant differences respect to the others clinical manifestations analysed.

Conclusions: There is a substantial overlap among different vasculitis, the presence or absence of some clinical and biological features can help in the differentiation and characterization of the different entitles.

KEY WORDS: Vasculitis. Clinical analysis.

Máiquez Asuero P, Abadín Delgado JA, Jiménez Plata C, Sánchez Romero A, Durán Quintana JA. Intoxicaciones digitálicas sospechadas y confirmadas. An Med Interna (Madrid) 2003; 20: 461-465.

\section{INTRODUCCIÓN}

El término vasculitis engloba a un grupo heterogéneo de enfermedades con el rasgo anatomopatológico común de presentar infiltrados inflamatorios en la pared de los vasos sanguíneos (1-5). Cualquier tipo de vaso puede estar lesionado, habiendo sido reconocidos un número limitado de lesiones histopatológicas. En general existe cierta correspondencia entre el calibre y la localización de los vasos afectados con los diferentes tipos de vasculitis descritos. Sin embargo, esta relación no siempre es unívoca, produciéndose con frecuencia solapamiento clínico y patológico entre las distintas entidades (1). Debido al desconocimiento de su etiopatogenia exacta y a los factores anteriormente señalados, se han propuesto múltiples clasificaciones de las vasculitis continuamente en revisión (2-4). Clínicamente pueden afectar cualquier órgano, ya sea de

Trabajo aceptado: 7 de mayo de 2003 
manera aislada o en combinación. El pronóstico de estas entidades es muy variable, pudiendo ser enfermedades fatales que precisan un diagnóstico y tratamiento precoces (7-10).

En la práctica clínica con frecuencia existen problemas para su correcta clasificación, lo que conlleva retrasos en el diagnóstico y tratamiento. En este trabajo presentamos 91 pacientes con vasculitis de pequeño y mediano vaso, deteniéndonos en las diferencias clínicas y biológicas entre aquellas vasculitis con mejor y peor pronóstico.

\section{PACIENTES Y MÉTODOS}

Criterios diagnósticos: Se realizó un estudio retrospectivo de 91 pacientes mayores de 16 años diagnosticados de vasculitis en nuestro hospital o en consultas externas de atención especializada (enero de 1991 a marzo de 2001). En 65 casos (70,5\%) el diagnóstico se obtuvo mediante hallazgos histológicos característicos junto con clínica compatible, siguiendo en todos los supuestos, menos en uno, las directrices de la "Conferencia de Consenso de Chapel Hill (CCCH)" sobre la nomenclatura de las vasculitis (3). En cuanto a aquellas con diagnóstico de "vasculitis por hipersensibilidad" (VH) (77 en total), incluyendo aquellas con este diagnóstico pero sin biopsia (25 casos), se siguieron los criterios de clasificación de la American College of Rheumatology de 1990 (2). Esta distinción se realizó en base a los criterios restrictivos que empleó la $\mathrm{CCCH}$, donde no se utiliza el término VH sino el de "vasculitis cutánea leucocitoclástica", quedando excluidos aquellos casos con afectación sistémica. Para el diagnóstico de "púrpura de Schönlein Henoch" (SH) se siguieron los criterios diagnósticos propuestos por Michael et al, o bien, éste se realizó en base a los resultados de la biopsia cutánea cuando se demostraba vasculitis con depósitos de IgA (6). Estos pacientes fueron atendidos y seguidos según los casos, tanto en régimen de hospitalización como en consulta, por los servicios de Dermatología, Medicina Interna, Nefrología o Reumatología. Criterios de exclusión: Se revisaron un total de 156 historias, de las que se excluyeron 65. De ellas, 10 se excluyeron por no tener biopsia realizada y no ser clasificables como vasculitis. El resto (55) eran vasculitis clasificables, aunque en pacientes menores de 16 años. La mayoría (53) correspondían a SH. Definiciones clínicas: Se consideró que la aparición de la vasculitis podría estar en relación con algún fármaco, cuando había una relación temporal con la administración de este (< 21 días desde el principio de los primeros síntomas). Cuando un paciente tenía clínica de infección respiratoria y recibió tratamiento con uno o más fármacos, ambos fueron considerados como posibles precipitantes. La púrpura palpable con biopsia positiva para vasculitis leucocitoclástica en pacientes con colagenosis previas o neoplasias fueron consideradas como VH $(4,9)$. Ello es debido a que probablemente el mecanismo patogénico en todas ellas es similar, en relación con la formación de depósitos inmunes en los vasos (4). La afectación renal se definió como la presencia de alteraciones en el sedimento de orina (hematuria y/o proteinuria) o la presencia de insuficiencia renal de forma secundaria al proceso. La afectación pulmonar se definió como cualquier afectación a este nivel en relación directa con la vasculitis y excluyendo procesos infecciosos o tumorales. Se consideró que la enfermedad había cursado con varios brotes cuando en el periodo comprendido desde el diagnóstico a la revisión de la historia
(2001) se había observado una reagudización, prolongación o falta de resolución del proceso. Se consideró que la mortalidad era debida a la vasculitis cuando era debida directamente a ella o secundaria a alguna complicación directamente relacionada (infección, isquemia). La mortalidad total fue la muerte del sujeto por cualquier causa. Valores analíticos: Solo se consideraron aquellos que podrían ayudar a diferenciar entre los distintos tipos de vasculitis (1). Se determinaron los valores de anticuerpos anticitoplasma de los neutrófilos (ANCA) en 36 casos (39,5\%), eosinófilos en sange en 79 $(86,8 \%)$, crioglobulinas séricas en $32(35,1 \%)$, anticuerpos antinucleares en $52(57,1 \%)$, serología para hepatitis C en 36 $(39,5 \%)$ y serología para hepatitis B en $44(48,3 \%)$. Estadísti$c a$ : las variables cualitativas se cuantificaron mediante número total de casos y proporciones simples. Se utilizó el Chi (2) y la prueba exacta de Fischer para comparar las proporciones de aparición de los distintos síntomas y afectaciones orgánicas a estudio entre los pacientes clasificados dentro de vasculitis con mecanismos patogénicos diferentes. En el primer bloque se agruparon aquellas en las que se ha involucrado el depósito de inmunocomplejos en los vasos [vasculitis por hipersensibilidad, púrpura de Schönlein-Henoch (SH), vasculitis crioglobulinémica esencial (CE)] y en el segundo aquellas en las que se han observan escasos depósitos inmunes (pauci-inmunitarias) y/o granulomas [poliarteritis nudosa $(\mathrm{PN})$, poliangeitis microscópica (PM), síndrome de Churg- Strauus (CS) y granulomatosis de Wegener $(\mathrm{GW})]$.

\section{RESULTADOS}

Frecuencia de los distintos tipos de vasculitis: En la tabla I se expone por orden de frecuencia los distintos tipos de vasculitis encontrados. Un caso con crioglobulinas positivas fue clasificado como PM por presentar un cuadro clínico y anatomopatológico compatible, junto con ANCA positivos. Datos antropométricos y antecedentes: De la muestra revisada el $57 \%$ eran varones y la edad media de todo el grupo de 61,9 \pm 18,6 años (mínima de 17 y máxima de 90). La edad media (en años) de los tipos de vasculitis más frecuentes fue: VH (63 \pm $19)$, SH $(53 \pm 23)$ y PM $(60 \pm 16)$. Referían infección respiratoria 21 casos $(23 \%)$ y posible relación con la toma de algún fármaco $25(27 \%)$. De los anteriores, 9 casos $(9,8 \%)$ referían los dos antecedentes. En $9(9,8 \%)$ coexistía una colagenopatía previa ya diagnosticada, en 8 sujetos $(8,7 \%)$ una neoplasia conocida y en $3(3,3 \%)$ otras infecciones ( 2 con hepatitis $\mathrm{C}$ que presentaban crioglobulinas negativas y 1 con paludismo). Un paciente con colagenosis refería infección respiratoria previa, otro ingesta sospechosa de fármacos y 4 pacientes con neoplasia conocida referían ingesta sospechosa previa de fármacos. Clínica asociada: En la tabla I se señalan por orden de frecuencia, los síntomas y órganos afectados en el conjunto total de la muestra. En la tabla II se desglosan los síntomas y órganos afectados según el tipo de vasculitis. En la tabla III se comparan las características clínicas encontradas entre las vasculitis cuyo mecanismo patogénico predominante parece ser por inmunocomplejos y aquellas en las que se encuentran escasos depósitos inmunitarios en la biopsia de tejidos o pauci-inmunitarias. En estas últimas se observa una proporción significativamente mayor de astenia, afectación multiorgánica y tendencia a la cronicidad. Excepto por la afectación pulmonar, no se observan síntomas u otras afectaciones orgánicas 
TABLA I

TIPOS DE VASCULITIS, SÍNTO MAS Y EVOLUCIÓN DE LA ENFERM EDAD EN EL CONJUNTO DE LA MUESTRA

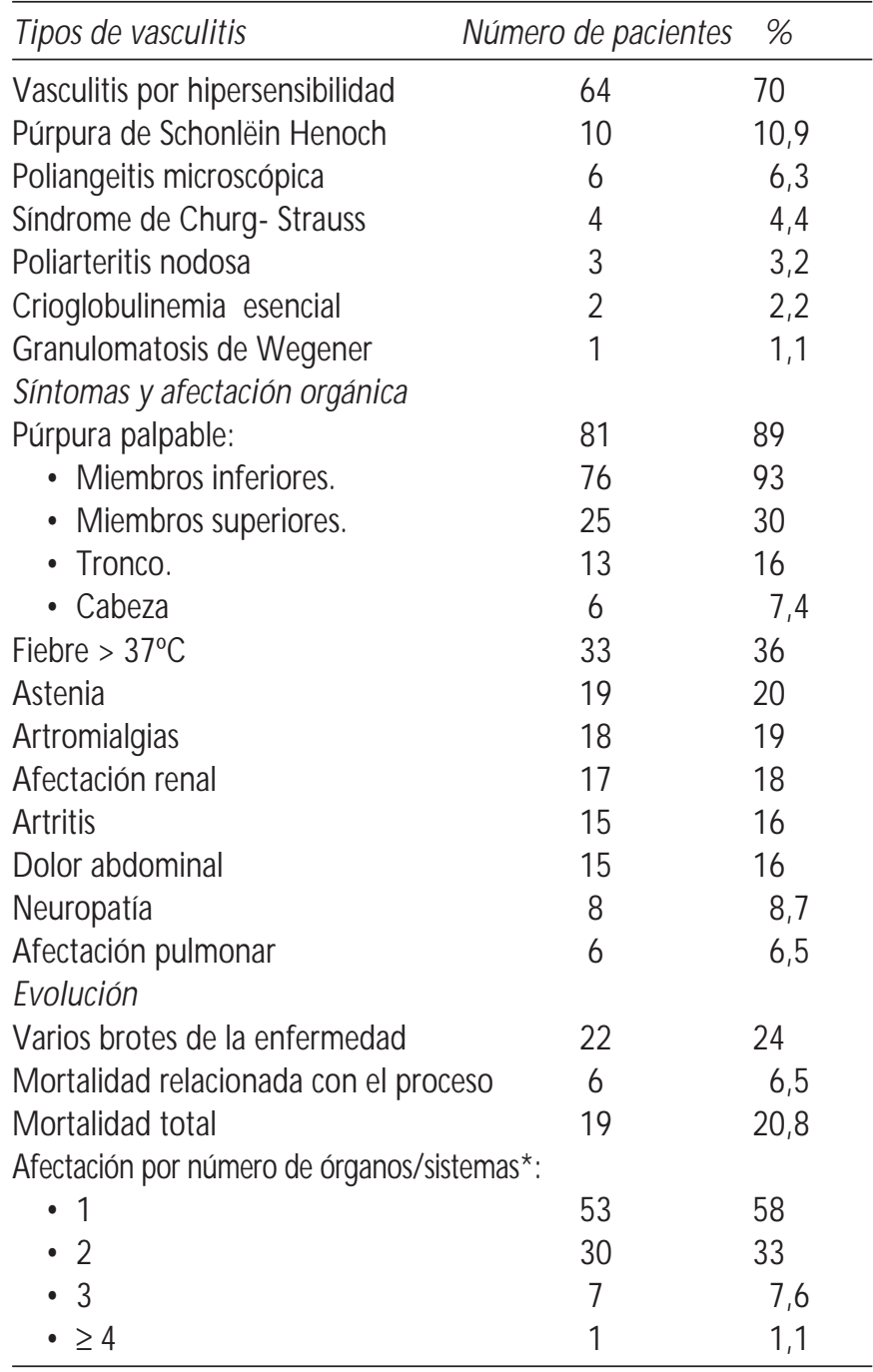

a: Se consideraron distintos órganos/ sistemas la afectación cutánea, articular, abdominal, renal, pulmonar y sistema nervioso.

que excluyan alguno de los dos bloques patogénicos planteados. 19 pacientes mostraban una $\mathrm{VH}$ en posible relación con fármacos, sin colagenopatía o neoplasia asociada. La frecuencia de síntomas asociados por orden de importancia en este subgrupo fue: fiebre $8(42 \%)$, artromialgias $1(5 \%)$, artritis 4 $(21 \%)$, dolor abdominal $1(5 \%)$, afectación renal $1(5 \%)$, afectación pulmonar y neuropatía $0(0 \%)$. Biopsias: Se obtuvo biopsia cutánea (57 casos), renal (10 casos), pulmonar (1 caso), nervio (1 caso), hepática (1 caso) y tabique nasal (1 caso). En tres pacientes se realizaron biopsias de distintos órganos. De las 66 biopsias realizadas, $65(98,4 \%)$ mostraban alteraciones anatomopatológicas sugestivas de vasculitis. Se observó vasculitis leucocitoclástica en 52 casos $(78,7 \%)$, necrosis fibrinoide en 9 (13,6\%), depósitos de IgA en 3 casos $(4,5 \%)$ y granuolmas necrotizantes en 1 caso $(1,5 \%)$. Todos los casos de vasculitis sin afectación cutánea fueron diagnosticados mediante biopsia. Resultados analíticos: Tomando como referencia el número de casos en los que se habían reali- zado las determinaciones analíticas ya citadas, la proporción de muestras positivas fue: ANCA $6(16,6 \%)$, eosinofilia 10 $(12,6 \%)$, crioglobulinas en sangre $3(9,3 \%)$, ANA $4(7,6 \%)$, serología positiva para hepatitis C $2(5,5 \%)$ y serología positiva de haber padecido hepatitis B $7(15,95) ; 3$ de ellos con antígeno positivo para este virus. Los casos positivos de ANCA se obtuvieron únicamente en aquellos casos con PM. Tratamiento y evolución: Recibieron corticoides 65 casos $(71,4 \%)$, antiinflamatorios no esteroideos $6(6,5 \%)$, inmunosupresores $15(16,4 \%)$, tratamientos tópicos cutáneos $16(17,5 \%)$, tratamiento antibiótico por infecciones intercurrentes $15(16,4 \%)$ y ningún tipo de tratamiento $14(15,3 \%)$. Los datos evolutivos figuran en la tabla 1. De los 19 pacientes con VH que recibieron algún fármaco sospechoso y no mostraban una colagenopatía o neoplasia subyacente, $3(15 \%)$ presentaron varios brotes de la enfermedad ( $18 \%$ consideradas todas las $\mathrm{VH}$; resultados no significativos).

\section{DISCUSIÓN}

La VH es la entidad más frecuentemente descrita en todas las series de vasculitis (6-9). La nomenclatura de este tipo de vasculitis es diversa y con frecuencia confusa. Los términos de vasculitis inducida por fármacos, vasculitis leucocitoclástica, vasculitis cutánea, vasculitis alérgica y otros, se han utilizado indistintamente y con frecuencia de forma inapropiada (6-9). En 1990 la American College of Rheumatology proponía cinco criterios para la clasificación de la "vasculitis por hipersensibilidad": edad mayor de 16 años, ingesta de un fármaco en relación temporal con los síntomas y con posibilidad de estar en relación con el cuadro, presencia de púrpura palpable o de rash maculopapular y, biopsia cutánea con neutrófilos alrededor de areterias o venulas. La presencia de tres o más de estos criterios muestra una sensibilidad y especificidad para el diagnóstico de "vasculitis por hipersensibilidad" del 71\% y $84 \%$ respectivamente (2). En este término también ha sido incluido por varios autores la púrpura de Schönlein Henoch y la Crioglobulinemia esencial, aunque estos criterios no distinguen entre la primera y aquella, siendo precisa la aplicación de criterios modificados o la presencia de IgA en la biopsia cutánea para su diferenciación $(6,8)$. Dependiendo de la distintas clasificaciones, la $\mathrm{VH}$ puede englobar a la vasculitis leucocitoclástica que se produce en el seno de una enfermedad inmunitaria o neoplásica (4). Ya que comúnmente se acepta que comparten un mecanismo patogénico similar, consideramos más apropiado clasificarlas bajo el mismo epígrafe.

La "vasculitis por hipersensibilidad" representa posiblemente un proceso por formación de inmunocomplejos circulantes o in situ, donde la expresión anatomopatológica cutánea más frecuente es la vasculitis leucocitoclástica (4). Se ha puesto en relación con la exposición a antígenos de diferente naturaleza como fármacos, componentes microbianos y productos tumorales. La VH se ha asociado a la ingesta de fármacos desde el 10\% hasta el 50\% de los casos, dependiendo de las series $(2,7,8)$. También se asocia a enfermedades sistémicas de carácter inmunitario (1) e infecciones agudas banales (principalmente respiratorias) e infecciones crónicas (7). Sin embargo en la mayoría de las series se desconoce el factor desencadenante, tal como ocurre en más de la mitad de nuestros pacientes. Generalmente es un proceso benigno, limitado a la piel y recortado, pero con cierta frecuencia recidiva o se 
TABLA II

SÍNTO MAS Y AFECTACIÓN O RGÁNICA SEGÚN LOS DISTINTOS TIPOS DE VASCULITIS

\begin{tabular}{lccccccc}
\hline & VH (64) & SH (10) & PM (6) & CS (4) & PN (3) & CE (2) & GW (1)* \\
\hline Púrpura palpable: & $64(100)$ & $10(100)$ & $1(16)$ & $3(75)$ & $0(0)$ & $2(100)$ & $0(0)$ \\
Fiebre > 37 oC & $21(32,8)$ & $4(40)$ & $3(50)$ & $2(50)$ & $2(66)$ & $0(0)$ & $0(0)$ \\
Posible relación con algún fármaco & $22(34,3)$ & $1(10)$ & $0(0)$ & $0(0)$ & $0(0)$ & $2(100)$ & $0(0)$ \\
Infección respiratoria previa & $15(23,4)$ & $2(20)$ & $0(0)$ & $1(25)$ & $1(25)$ & $0(0)$ & $0(0)$ \\
Astenia & $9(14,0)$ & $1(10)$ & $2(33)$ & $3(75)$ & $3(100)$ & $1(50)$ & $0(0)$ \\
Artromialgias & $9(14,0)$ & $4(40)$ & $2(33)$ & $2(50)$ & $1(33)$ & $0(0)$ & $0(0)$ \\
Afectación renal & $2(3,1)$ & $6(60)$ & $6(100)$ & $0(0)$ & $4(100)$ & $0(0)$ & $0(0)$ \\
Artritis & $11(17,1)$ & $3(30)$ & $0(0)$ & $0(0)$ & $0(0)$ & $0(0)$ & $1(100)$ \\
Dolor abdominal & $4(6,2)$ & $6(60)$ & $2(33)$ & $0(0)$ & $2(66)$ & $1(50)$ & $0(0)$ \\
Neuropatía & $4(6,2)$ & $0(0)$ & $0(0)$ & $2(50)$ & $1(33)$ & $1(50)$ & $0(0)$ \\
Asma & $0(0)$ & $0(0)$ & $0(0)$ & $3(75)$ & $0(0)$ & $0(0)$ & $0(0)$ \\
Afectación pulmonar & $0(0)$ & $0(0)$ & $3(50)$ & $3(75)$ & $0(0)$ & $0(0)$ & $0(0)$ \\
Varios brotes de la enfermedad & $11(17,1)$ & $3(30)$ & $4(66)$ & $3(75)$ & $2(66)$ & $0(0)$ & $0(0)$ \\
M ortalidad relacionada con el proceso & $0(0)$ & $2(20)$ & $2(33)$ & $0(0)$ & $2(66)$ & $0(0)$ & $0(0)$ \\
M ortalidad total & $10(15,6)$ & $4(40)$ & $2(23)$ & $1(25)$ & $2(66)$ & $0(0)$ & $0(0)$ \\
Afectación 2 2 órganos/ sistemas & $16(25)$ & $9(90)$ & $5(83)$ & $4(100)$ & $2(66)$ & $1(50)$ & $1(100)$ \\
\hline
\end{tabular}

* varón de 67 años con epíxtasis, úlceras nasales, perforación de tabique, artritis, pANCA positivos y biopsia de tabique nasal compatible con vasculitis con necrosis fibrinoide; H: Vaculitis por hipersensibiulidad; SH: Púrpura de Schonlëin Henoch; PM : Poliangeitis microscópica; CS: Síndrome de Churg- Strauss; PN: Poliarteritis nodosa; CM E; Crioglobulinemia esencial; GW; Granulomatosis de Wegener. Se expresa en número total de pacientes (porcentaje).

\section{TABLA III}

COM PARACIÓ N DE LOS PARÁM ETROS CLÍNICOS ENTRE LAS VASCULITIS POR DEPÓ SITO DE INMUNO COM PLEJOSY AQUELLAS PAUCI-INM UNITARIAS.

\begin{tabular}{|c|c|c|}
\hline & $\begin{array}{l}\text { pósitos inmunes } \\
\text { en los vasos } \\
\quad(n=77)\end{array}$ & $\begin{array}{c}\text { Pauci-inmunitarias y/o } \\
\text { granulomas } \\
(n=14)\end{array}$ \\
\hline Edad (años) & $62 \pm 19$ & $60 \pm 13$ \\
\hline Varones & $42(55)$ & $10(71)$ \\
\hline $\begin{array}{l}\text { Infección respiratoria } \\
\text { previa }\end{array}$ & $19(25)$ & $2(14)$ \\
\hline $\begin{array}{l}\text { Posible relación con } \\
\text { fármacos }\end{array}$ & $24(31) c$ & $0(0)$ \\
\hline Púrpura palpable & $77(100) d$ & $4(29)$ \\
\hline Fiebre $>37^{\circ} \mathrm{C}$ & $26(34)$ & $7(50)$ \\
\hline Astenia & $11(14) d$ & $8(57)$ \\
\hline Artromialgias & $13(17)$ & $5(36)$ \\
\hline Dolor abdominal & $11(14)$ & $4(29)$ \\
\hline Artritis & $14(18)$ & $1(7)$ \\
\hline Neuropatía & $5(6)$ & $3(21)$ \\
\hline Afectación renal & $8(10) d$ & $9(64)$ \\
\hline Afectación pulmonar & $0(0) e$ & $6(43)$ \\
\hline Afectación $\geq 2$ & $26(34) d$ & $12(86)$ \\
\hline órganos/ sistemas & 14 (18)e & $8(57)$ \\
\hline \multicolumn{3}{|l|}{ Varios brotes } \\
\hline $\begin{array}{l}\text { M ortalidad relacionada } \\
\text { con el proceso }\end{array}$ & $2(2,6) c$ & $4(28)$ \\
\hline M ortalidad total & $14(18)$ & $5(36)$ \\
\hline
\end{tabular}

a: Predominantemente cutánea, Púrpura de Schonlëin Henoch, Crioglobulinemia esencial. b: Síndrome de Churg- Strauss, Poliangeitis microscópica, Poliarteritis nodosa. c: $p<0,05$. d: $p<0,001$. e: $p<0,01$. Expresado en número de pacientes $(\%)$. cronifica, y ocasionalmente asocia diversa afectación visceral $(4,6,8)$. Los parámetros evolutivos, manifestaciones sistémicas y complicaciones viscerales de nuestra serie no difieren de los descritos, a pesar del sesgo de selección de la muestra, por provenir ésta de un medio hospitalario y de atención especializada. En nuestros pacientes con $\mathrm{VH}$ se ha observado una mortalidad total mayor de la esperada en relación con la inclusión de pacientes con colagenosis y neoplasias subyacentes.

La presencia de púrpura cutánea palpable es un dato clínico prácticamente patognomónico de vasculitis de pequeño vaso, aunque no específico de las vasculitis por hipersensibilidad (1,9). Su presencia, como se demuestra en nuestra serie, se observa en otras vasculitis sistémicas en las que se han involucrado otros mecanismos etiopatogénicos (5). Otros síntomas como la astenia, anorexia, artromialgias, artritis, fiebre y dolor abdominal, pueden superponerse entre los distintos tipos de vasculitis, sin ser en general de gran valor en el diagnóstico diferencial entre estas entidades (1). Entre nuestros pacientes únicamente la astenia apareció de forma significativamente mayor entre los que presentaban vasculitis de peor pronóstico. Observamos que la mayor afectación visceral, número de órganos o sistemas implicados y la cronificación del proceso pueden hacer sospechar clínicamente la presencia de este tipo de vasculitis, aunque no descartan una VH. Estos datos concuerdan con los ya publicados, mostrándonos la poca relevancia de la sintomatología descrita en el contexto de estas enfermedades $(1,2)$.

Varios son los mecanismos patogénicos descritos, y no mutuamente excluyentes, implicados en el desarrollo de las vasculitis. Entre ellos destacan el depósito de complejos inmunes en los vasos (ya citado), la producción de anticuerpos anticitoplasma de los neutrófilos (ANCA), la producción de anticuerpos anticélula endotelial y una respuesta inmunológica mediada por células $\mathrm{T}$ frente a antígenos presentes en la 
pared de los vasos (1). Los ANCA son anticuerpos dirigidos contra ciertas proteinas del citoplasma de estas células. Se encuentran en elevado porcentaje en los pacientes con vasculitis sistémicas en los que no se encuentran depósitos inmunes en los vasos (10). Generalmente identifican aquellas con peor pronóstico, y por tanto, donde es más importante adoptar un tratamiento precoz $(1,5)$. En nuestra serie ninguno de los pacientes clasificados en la categoría de vasculitis por hipersensibilidad en los que se realizó esta determinación, mostró la presencia de ANCA. Su ausencia en aquellos casos con púrpura cutánea aislada ofrece escasa sensibilidad (11), pero su presencia obliga al clínico a una vigilancia más estrecha del paciente y a descartar con mayor profundidad una afectación multiorgánica (12). Teniendo en cuenta los resultados observados y de acuerdo con otros autores, creemos oportuno la realización de estas pruebas desde fases precoces en todos los enfermos con vasculitis (1).

\section{Bibliografía}

1. Blanco R, Martinez-Taboada VM, Rodríguez-Valverde V, GarcíaFuentes M. Cutaneous vasculitis in children and adults: Associated diseases and etiology factors in 303 patients. Medicine (Baltimore) 1998; 77: 403-11

2. Calabrese LH, Duna GF. Drug-induced vasculits. Curr Opin Rheum 1996; 8: 34-40

3. Cupps TR, Fauci AS. The vasculitis syndromes. En: Cupps TR, Fauci AS, eds. Advances in internal medicine. Chicago: Year Book Medical pub Inc 1982;27: 315-44

4. Daoud MS, Gibson LE, DeRemee RA, et al. Cutaneous Wegener's granulomatosis: Clinical, histopatologic and immunopathologic features of thity patients. J Am Acad Dermatol 1994; 31: 605-10.

5. Hagen EC, Daha MR, Hermans J et al.. Diagnostic value of standardized assays for anti-neutrophil cytoplasmatic antibodies in idiopatic systemic vasculitis. EC/BCR Project for ANCA Assay Standardization. Kidney Int 1998; 53: 743-53.

6. Hunder GG, Arend WP, Bloch DA, et al. The American College of Rheumatology 1990 criteria for the classification of vasculitis. Arthritis Rheum 1990; 33: 1065-7.

7. Jannette JC, Falk RJ, Andrassy K, et al. Nomenclature of systemic vasculitides. Proposal of an international consensus conference. Arthritis Rheum 1994; 37: 187-92.

8. Jennette JC, Falk RJ. Small-vesel vasculitis. N Engl J Med 1997; 337: $1512-23$
La presencia y ausencia de otros datos de laboratorio y anatomopatológicos serán claves en el diagnóstico diferencial de estas entidades1. Además de los ANCA, en esta categoría se incluyen la presencia de depósitos por inmunocomplejos IgA o granulomas en las muestras de biopsia, las crioglobulinas séricas o en vasos, y la asociación de asma y eosinofilia1. La presencia de crioglobulinas en sangre identificó aquellos dos pacientes con una "Crioglobulinemia mixta esencial", los cuales asociaban una infección crónica por hepatitis C (13). La presencia de granulomas necrotizantes se puede observar en la GW o en el CS, pero no se observa en las otras entidades (14). La afectación del aparato respiratorio es frecuente en las vasculitis con ANCA positivos, pero es rara en aquellas con patogenia relacionada con inmunocomplejos $(15,16)$. En nuestra serie ningún caso de este grupo de vasculitis presentaba asma o afectación pulmonar. Su presencia, por lo tanto, nos alertará sobre aquellas vasculitis con peor pronóstico (15).

9. Jennette JC, Wilkman AS, Falk RJ. Diagnostic predictive value of ANCA serology. Kidney Int 1998; 53: 796-99.

10. Lhote F, Guillevin L. Polyarteritis nodosa, microscopic polyangiitis, and Churg-Strauus syndrome: clinical aspects and treatment. Rheum Dis Clin North Am 1995; 21: 911-47.

11. Martínez-Taboada VM, Blanco R, García M, Rodríguez V. Clinical features and Outcome of 95 Patientes with Hypersensitivity vasculitis. Am J Med 1997; 102: 186-91.

12. Michel BA, Hunder GG, Bloch DA, Calabrase LH. Hypersensitivity vasculitis and henoch-Schönlein purpura: A comparison between the 2 disorders. J Rheumatol 1992; 19: 721-728.

13. Monti G, Galli M, Invernizzi F, et al. Cryoglobulinemias: a multi-centre study of the early clinical and laboratory manifestations of primary and secundary disease. GISC. Italian group for the Study of Cryoglobulinemias. QJMed 1995; 88: 115-126.

14. Niles JL, Bottinger EP, Saurina GR, et al. The syndrome of lung hemorrhage and nephritis is usually an ANCA-associated condition. Arch Intern Med 1996; 156: 440-50.

15. Sáez-Royuela A, Hernández-Jaras J, Calvo C, et al. Vasculitis sistémica con afectación renal grave: descripción de 18 casos. An Med Interna (Madrid) 1998; 15: 311-5.

16. Savige J, Davies D, Falk RJ, Jennette JC, Wiik A. Antineutrophil cytoplasmic antibodies and associated diseases: a review of the clinical and laboratory features. Kidney Int 2000; 57: 846-62. 